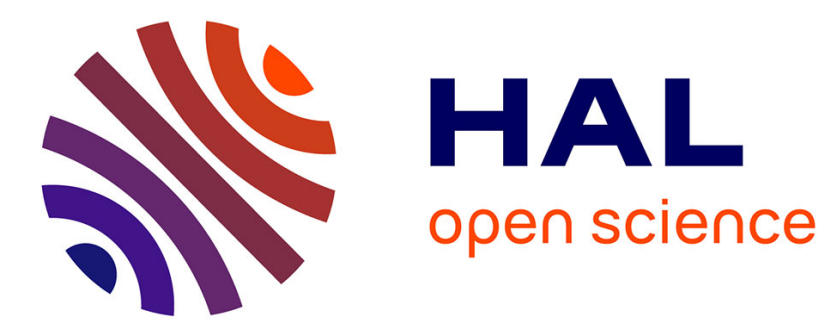

\title{
Le réseau personnel de gestion des connaissances et la redéfinition $\mathrm{du}$ travail
}

\author{
Olivier Le Deuff
}

\section{To cite this version:}

Olivier Le Deuff. Le réseau personnel de gestion des connaissances et la redéfinition du travail. Terminal. Technologie de l'information, culture \& société, 2012, 110, pp.39-54. sic_00776845

\section{HAL Id: sic_00776845 \\ https://archivesic.ccsd.cnrs.fr/sic_00776845}

Submitted on 16 Jan 2013

HAL is a multi-disciplinary open access archive for the deposit and dissemination of scientific research documents, whether they are published or not. The documents may come from teaching and research institutions in France or abroad, or from public or private research centers.
L'archive ouverte pluridisciplinaire HAL, est destinée au dépôt et à la diffusion de documents scientifiques de niveau recherche, publiés ou non, émanant des établissements d'enseignement et de recherche français ou étrangers, des laboratoires publics ou privés. 
Version preprint de l'article. Le Deuff, Olivier.(2012) Le réseau personnel de gestion des

connaissances et la redéfinition du travail, Terminal $\mathrm{n}^{\circ} 110$, p.39-54

\title{
Le réseau personnel de gestion des connaissances et la redéfinition du travail
}

\author{
Olivier Le Deuff. Docteur en sciences de l'information et de la communication
}

\author{
LaboratoireMICA.EA 4426 Université de Bordeaux 3 \\ Oledeuff@gmail.com
}

Nous souhaitons montrer que la démarche du PKM (personalknowledge management) constitue une nouvelle manière de considérer l'organisation des connaissances ainsi que la définition habituellement conférée au travail. Nous n'avons donc pas l'intention deconforter l'idée d'unemort imminente $^{1} \mathrm{du}$ Knowledge Management (KM) mais plutôt d'esquisser de nouvelles manières d'envisager son déploiement et son évolution.La gestion des connaissances ou Knowledge Management se rapporte aux méthodes et outils mis en place pour favoriser la conservation, le repérage et la diffusion d'informations, de savoirs et de savoir-faire au sein d'une organisation.L'objectif étant pour l'organisation de parvenir à obtenir un avantage économique via des transferts de connaissances optimisés. (Grant, 1996) Le $P K M$ situe l'approche d'abord à l'échelon individuel (Pollard, 2008) comme élément moteur du partage des connaissances.

En effet,le knowledge management ne peut demeurer dans une approche de type top-down imposée de manière hiérarchique mais doit au contraire davantage reposer sur l'échelon individuel dans une construction bottom-up. La position inverse a longtemps prédominé avec l'influence des travaux des japonais Takeuchi et Nonaka (Nonaka, 2006). Cependant, les écrits des japonais visaient principalement à décrire une structure collective qui montait en puissance : l'entreprise japonaise et à travers elle, un nouveau modèle qui prétendait au leadership mondial espérant l'entrée dans un nouveau cycle marquant le déclin des Etats-Unis et la montée en puissance du Japon et ce dans la lignée de l'ouvrage de Paul Kennedy (Kennedy, 2006).

Nous proposons donc d'examiner d'abord dans une première section les raisons de l'échec relatif du $K M$ pour montrer en quoi un système reposant sur l'échelon individuel à plus de chances de réussir en rappelant le contexte d'émergence des théories de la gestion des connaissances.Dans une seconde partie, nous montronsen quoi les outils de gestion avancée de l'information, notamment ceux dans la lignée du web 2.0, influent sur la définition de la gestion des connaissances et sur la manière dont peut se constituer le $P K M$. A cet effet, cette étude théorique s'accompagne d'une enquête en ligne auprès de 302 personnes volontaires que nous pouvons qualifier de «travailleurs du savoir et de l'information »(knowledgeworkers). Nous entendons par travailleurs du savoir des personnes qui utilisent quotidiennement des informations et des sources d'informations ${ }^{2}$ dans le cadre de leur travail. Les résultats présentés ici démontrent l'influence très nette des outils de gestion de flux d'informationsainsi que des outils utilisables en mode nomade. Nous consacrons la troisième section de cet article à la manière dont évoluent les représentations du travail en constatant le fait que la dichotomie travail/loisirs devient beaucoup moins nette.

\footnotetext{
${ }^{1}$ Sur le blog green Chameleon, il est possible de retrouver débats et réflexions autour de ce sujet<http://www.greenchameleon.com/gc/blog_detail/dead_km_walking/>

${ }^{2}$ Nous prenons ici le mot information dans son acception la plus large depuis la donnée jusqu'à la connaissance en passant par de l'information de type news.
} 


\section{Du $K M$ au PKM}

L'opposition entre connaissances tangibles et connaissances intangibles décrites pas Nonaka et Takeuchia parfois été quelque peu caricaturée à l'excès.Les chercheurs opposaient les connaissances intangibles et tacites, qui restent au stade de l'intuition, aux connaissances tangibles qui suivent un processus de rationalisation et qui peuvent être transmises via notamment des documents synthétiques ou explicatifs. L'objectif de la gestion des connaissances étant alors de veiller à transformer le maximum de connaissances intuitives en éléments de connaissance pouvant être transférés.

\subsection{Un contexte théorique négligé}

Les théories des japonais ont été adaptées plus ou moins de manière opportune et surtout en omettant totalement d'en repréciser le contexte.Trop souvent, il a été oublié que les recherches et conclusions des japonais s'appliquaient d'abord à un contexte national et que son application élargieau niveau international méritait d'importants réajustements. De même, le contexte initial d'élaboration correspond aussi à une période économique florissante pour le Japon avec une forte stabilité dans l'emploi. Cela permettait aussi d'idéaliser quelque peu un collectif surplombant qui prédominerait sur l'individu. L'injonction essentiellement hiérarchique marquait aussi la volonté d'imposer une stratégie globale minimisant les divergences individuelles avec le risque final de réduction del'investissement personnel. C'est toute la dimension duCHI japonais qui renvoie à une volonté supérieure et puissante qui est aussi la marque de cette forte dimension hiérarchique. Très souvent, l'application du modèle dans le reste de l'Occident s'est arrêtée à ce niveau.L'influence des travaux des japonais porte surtout sur leurs premières recherches or ils ont approfondi et amélioré sans cesse leurs modèles dont lefameux SECI (Socialisation, externalisation, combinaison et Intégration) avec la passage des connaissances tacites en connaissances tangibles et le modèle du $B a$ qui avait le mérite de prendre en compte une diversité de besoins et d'objectifs pas uniquement financiers. Cette prise de soin de l'individu au sein du collectif a souvent fait défaut dans les dispositifs qui ont été inspirés par ces théories.

Or le succès du KMrepose principalement surl'investissement de l'individu et son intérêt rationnel ainsi qu'affectif à participer à un processus collectif ; un processus qui nécessite une relation entre les représentationsindividuelles et l'espace commun de connaissances (commonknowledge) que définit Robert Grant (Grant, 1996) comme l'intersection des connaissances personnelles, des éléments de langage et de communication commune, des pratiques partagées et des objectifs collectifs.Initialement, le processusde gestion de connaissances peut paraitre parfoiscomme une forme de désappropriation de connaissances qui peut aboutir à une forme d'aporie bien connue du changement organisationnel : Pierre ne s'investira que si Paul commence à le faire, sauf que Paul a décidé de ne s'investir que si Pierre commence.

Le $K M$ devient alors un jeu de dupes où les craintes d'être perdant dans la donne sont des critères trop importants pour que le partage de connaissances soit réellement opérationnel.

Le climat économique constitue évidemment un frein important : une telle démarche ne peut être mise en place que dans un climat de confiance mutuelle. Un tel climat pouvait exister il y a encore quelques années dans les entreprises japonaises où le salarié bénéficiait d'un emploi quasi garantie à vie. Aujourd'hui, cette situation devient fort rare au niveau mondial, y compris d'ailleurs dans la fonction publique française où le rapport de confiance avec l'institution devient faible ce qui explique d'ailleurs bon nombre d'échecs de projets et de réformes notamment au sein de l'Education Nationale. 


\subsection{Définition du PKM}

Comment parvenir dès lors à mettre en place des stratégies collaboratives de partage de connaissances dans l'entreprise face à ces obstacles organisationnels, institutionnels, économiques, psychiques et ...individuels ? Le $P K M$ tente d'y répondre en commençant par poser les bases au niveau individuel. Il recoupe fortement des tendances actuelles notamment liées à la personnalisation de l'information qui se développent autour d'applications de type web 2.0 et s'inscrit dans la lignée des communautés de pratique (Wenger, 1998) et par ricochet des réseaux sociaux. L'individu est donc placé au centre du système du $K M$ comme le décrit parfaitement Christophe Deschamps :

"Ainsi au niveau personnel l'employé d'une organisation aura à gérer:

- Les données, informations et connaissances utiles à son travail quotidien

- Ses propres compétences dans le cadre de l'évolution de sa carrière et des bouleversements divers qu'elle ne manquera pas de connaître

- Le réseau personnel qu'il développe au long de sa carrière (et plus généralement de sa vie) » ${ }^{3}$

Ces trois aspects sont les éléments autour desquels se construit cette gestion des connaissances à l'échelon individuel.
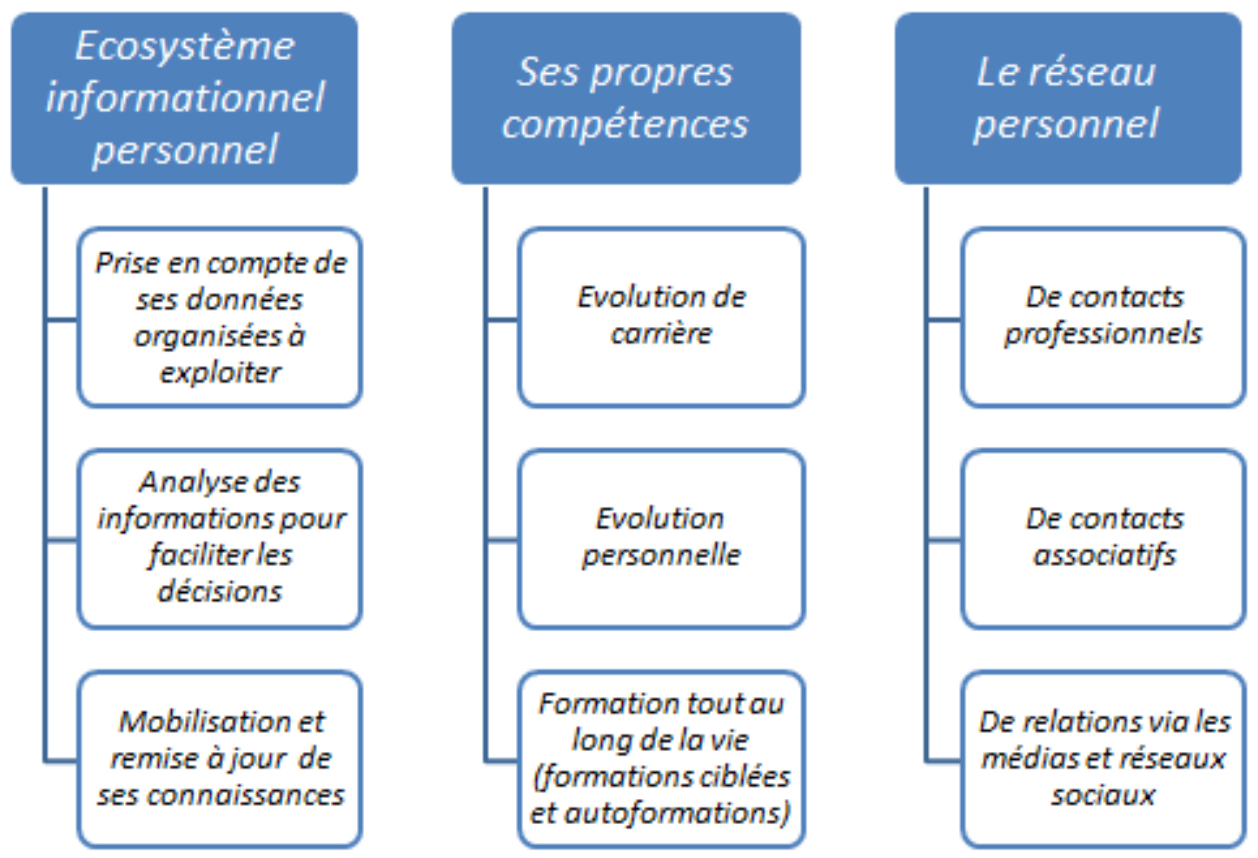

Le $P K M$ heurte parfois certains puristes qui considèrent que la gestion des connaissances ne peuts'inscrire que dans une démarche collective, il convient de rappeler que les principaux théoriciens du $K M$ comme Peter Drucker considéraient qu'il y avait bien un management de soi dans le management de la connaissance. KirbyWrightavait déjà bien démontré en 2005 l'intérêt de prendre en compte les performances individuelles des travailleurs dans le processus de gestion des

\footnotetext{
${ }^{3}$ Deschamps, C. Le PersonalKnowledge Management, ou comment mieux gérer vos informations personnelles <http://www.outilsfroids.net/news/le-personal-knowledge-management-ou-comment-mieux-gerer-vos-informationspersonnelles> in Outils froids. Décembre 2005
} 
connaissances (Wright, 2005).Il convient aussi désormais de prendre également en compte les objets techniques qu'utilisent et emploient le travailleur. Cet environnement de travail n'étant pas uniquement circonscrit à ce que lui met à disposition son employeur mais concernant la somme des outils qu'il mobilise pour remplir ses missions.

C'est pourquoi une des définitions les plus intéressantes du $P K M$ provient du journaliste et chercheur Steve Barth, spécialisé dans les domaines de l'intelligence collaborative :

«Le PersonalKnowledge Management met en jeu un ensemble de techniques et d'outils relativement simples et peu coûteux que chacun peut utiliser pour acquérir, créer et partager la connaissance, étendre son réseau personnel et collaborer avec ses collègues sans avoir à compter sur les ressources techniques ou financières de son employeur. » ${ }^{4}$

Concrètement, cela signifie que l'individu va travailler au sein de communautés de pratiques et de réseaux qui ne sont pas restreints à l'entreprise. L'individu apprend donc de lui-même à développerdes stratégies de partage de connaissances et de savoirs au sein de réseaux, notamment professionnels, de type communautés de pratiques, mais également de plus en plus interprofessionnels. Ces pratiques acquises personnellement peuvent être plus aisément réinvesties au sein de l'entreprise ou de l'organisme du fait d'une aisance acquise extérieurement. Mais le gain principal pour l'individu, c'est qu'il garde son indépendance vis-à-vis de l'entreprise. Le partage des connaissances s'effectue d'ailleurs principalement en dehors et s'avère moins cloisonné. L'individu peut gagner de par sa participation à ces réseaux une forme de légitimité et de compétences qu'il pourra éventuellement utiliser comme argument de négociation si cette dernière est reconnue au sein de l'entreprise, ce qui n'est pas toujours le cas. Néanmoins, l'individu n'aura pas l'impression de s'être «désapproprié » de ses connaissances mais aura au travers du PKM constitué un réseau au sein duquel il pourra, selon son niveau, puiser de l'information, obtenir conseils ou bonnes pratiques et tisser des relations professionnelles. Ces «connaissances » pourront lui servir personnellement mais également s'avérer également utiles pour l'entreprise qui pourra ainsi bénéficier d'une expertise et d'un réseau élargi.

En effet, la construction d'une gestion des connaissances à partir du développement des profils individuels n'est pas antinomique avec une construction collective, bien au contraire. C'est aussi rappeler l'importance de la médiation dans la transmission en considérant la connaissance autant de par son acceptation « savoir » que par son renvoi à la « ressource humaine ».

Cela implique toutefois une redéfinition des systèmes de gestion de connaissances et probablement de nouveaux modes de fonctionnement dans les organisations. Il convient de constater que bien souvent il y a une confusion entre la collaboration et le respect de la hiérarchie. Par conséquent, le fait de développer une gestion de connaissances à partir de l'individupeut faire craindre une remise en cause du système décisionnaire.Il est vrai que l'influence du web 2.0 ne peut qu'interroger, tant il est davantage basé sur des mécanismesbottom-up que top-down au point qu'il privilégie nettement la popularité à l'autorité (Le Deuff, 2009) ce qui ne peut qu'interroger les organisations sur leur culture de l'information. L'effet web 2.0 apparaît nettement influencé les pratiques informationnelles et les usages.

\section{Un PKM en développement : l'effet web 2.0}

Nous avons tenté de mesurer justement cet effet «web 2.0 » pour tenter de voir en quoi les outils de gestion avancée de l'information pouvaient constituer un effet de développement du PKM.

${ }^{4}$ La traduction est de Christophe Deschamps.Voir Deschamps, 2009. p.183 


\subsection{Méthodologie et objectifs de l'enquête}

Les résultats présentés ici s'appuient sur une enquête en ligne réalisée durant le mois de janvier 2011 auprès de 302 répondants dont 298 questionnaires complets et exploitables. Elle a été diffusée sur le web notamment sur des canaux tels que Twitter. Le public de l'enquête est donc essentiellement un public habitué à utiliser les différentes applications offertes par le web et le web 2.0. Nous avons cherché à déterminer si de nouvelles habitudes et pratiques se développaient en lien avec les environnements numériques. Il s'agissait de voir si la division travail/loisirs était nettement marquée et si les outils de gestion de l'information avaient une influence sur les manières de gérer son travail et son réseau personnel d'informations et de connaissances. Nous avons donc ciblévolontairement un public « spécialisé » qui utilise l'information de manière récurrente dans son travail. Ces «travailleurs du savoir » ayant répondus sont essentiellement des personnes exerçant dans les secteurs de la documentation, des bibliothèques, de l'enseignement, de la recherche, de l'informatique, de l'architecture de l'information, des DSI et du journalisme. Le public qui a répondu est essentiellement féminin (62\%) et la grande majorité a entre 25 et 45 ans (67,5\%). Il n'est donc pas pleinement représentatif des travailleurs de l'information et du savoir mais représente plutôt un public initié qui a alors le mérite de nous aider à anticiper de futures tendances.

Les technologies et leurs usages modifient les modes de communications et de transmission des informations et des connaissances dans les organisations. George Huber (Huber, 1990) signalait en 1990 que les technologies avancées de l'information présentaient d'ailleurs des aspects fort différents des techniques plus classiques et considérait qu'il fallait repenser les théories en matière d'organisation des connaissances en mesurant leur impact. Clairement, il faudrait procéder de même avec les outils issus du web 2.0 tels les agrégateurs de flux $R s s$, les pages d'accueil personnalisé, les signets sociaux et les réseaux sociaux. Ils ont eu un impact évident sur les pratiques et les usages des personnes ayant répondu à notre enquête. Un grande majorité des répondants constatent et admettent une influence dans leur travail quotidien et leurs méthodes de gestion de leur information (figure 1). Des réflexions avaient été déjà menées au sujet de l'apport des outils collaboratifs tels les wikis dans le management des connaissances en entreprise (Ranman, 2006 ; Wagner, 2004).Ranman notait que l'outil wiki était riche en potentialités mais que son usage insuffisamment intuitif limitait de beaucoup sa portée. Wagner soulignait que le wiki nécessitait une organisation et des formes de régulation. Cette complexité constatée amène d'ailleurs son concepteur Ward Cunningham à faire évoluer le dispositif actuellement. Des outils plus récents, comme les agrégateurs de flux rss, présentent parfois des éléments de motivation plus visible et des retours plus immédiats pour l'individu que ne le permet le wiki.Ces outils aisés d'utilisation en apparence nécessitentcependant de réels apprentissages voire de véritables formations dédiées. 


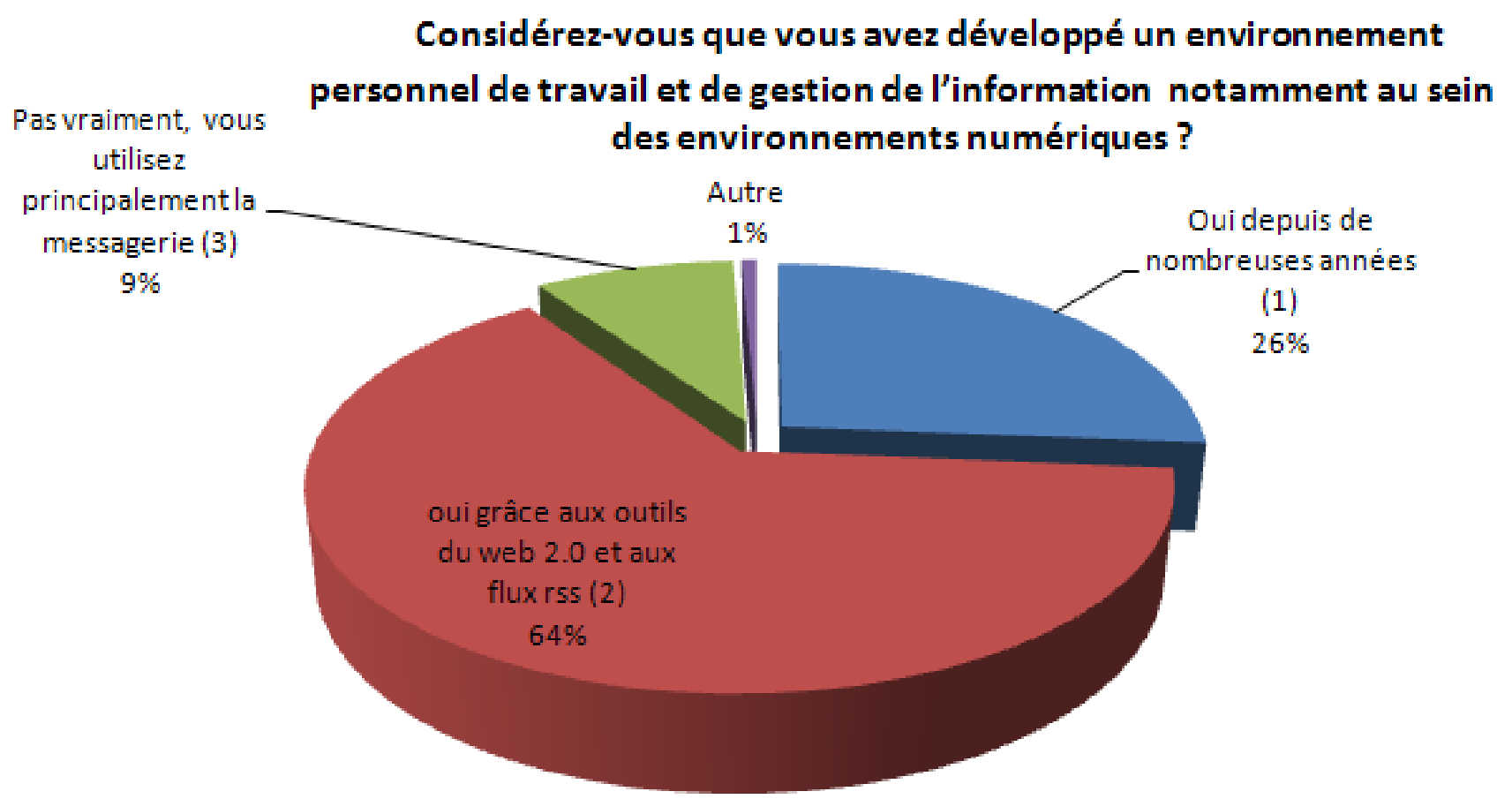

Figure 1. La constitution d'un environnement numérique du travail.

L'utilisation des flux rssest un des outils le plus emblématique de ce changement en étant plébiscité (figure 2). Il semble donc devenu un outil indispensable à toute personne souhaitant effectuer une veille régulière. C'est aussi un mode d'appropriation de la somme d'informations circulant sur les réseaux avec une stratégie organisée.Le réflexe semble avoir remplacé parfois la lecture du quotidien. Un retour en arrière semble même impossible selon un professionnel ayant répondu à cette enquête :

«Je ne travaille plus comme avant et je ne pourrai plus travailler comme y a 3 ans en arrière. »

\section{Utilisez-vous un agrégateur de flux}

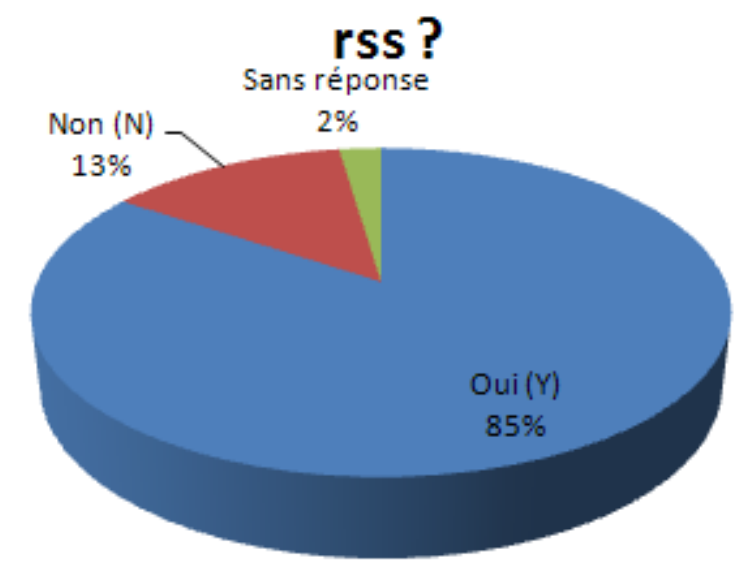

Fig. 2 L'utilisation des agrégateurs de flux rss.

Le caractère souvent indispensable et fort utile souligné contraste aussi avec l'impression que les gains de temps ne sont pas au rendez-vous dans le sens où l'information suscite sans cesse de 
nouveaux besoins d'information et de formation. De plus, beaucoup considèrent qu'il faut dès lors se construire en sus de l'environnement technique, une stratégie cognitive qui permet d'éviter les effets de mode :

"La difficulté est de distinguer ce qui reste de l'effet de mode (et qui disparaîtra au bout de quelques mois), par rapport à de véritables concepts et outils "durables" et fédérateurs. »

Il reste aussi que certains constatent le caractère envahissant de ces environnements de par leur côté convivial voire ludique:

"L'information devient en elle-même parfois quelque chose de terriblement "ludique". C'est pas pour rien qu'on parle d'infotainement! Il y a (de ma part) encore un gros travail à faire pour distinguer l'information consommation de l'information qui peut avoir un apport critique dans mon cadre professionnel. »

Cette intrusion du travail dans la sphère des loisirs voire sa dissolution se fait sentir. Un professionnel de notre enquête évoque même la disparition progressive de cette frontière : "Plus de travail à la maison, moins de temps libre, moins de frontière entre les deux »

Nous avons tenté une visualisation avec l'outil wordle des réponses à la question ouverte sur les changements perçus ${ }^{5}$. Plusieurs éléments nous paraissent intéressants à commenter.

${ }^{5}$ La question exacte était la suivante : Remarques libres et réflexions sur les changements dans votre manière de travailler et de gérer votre carrière par rapports aux environnements informationnels notamment depuis l'apparition des outils dans la lignée du web 2.0 


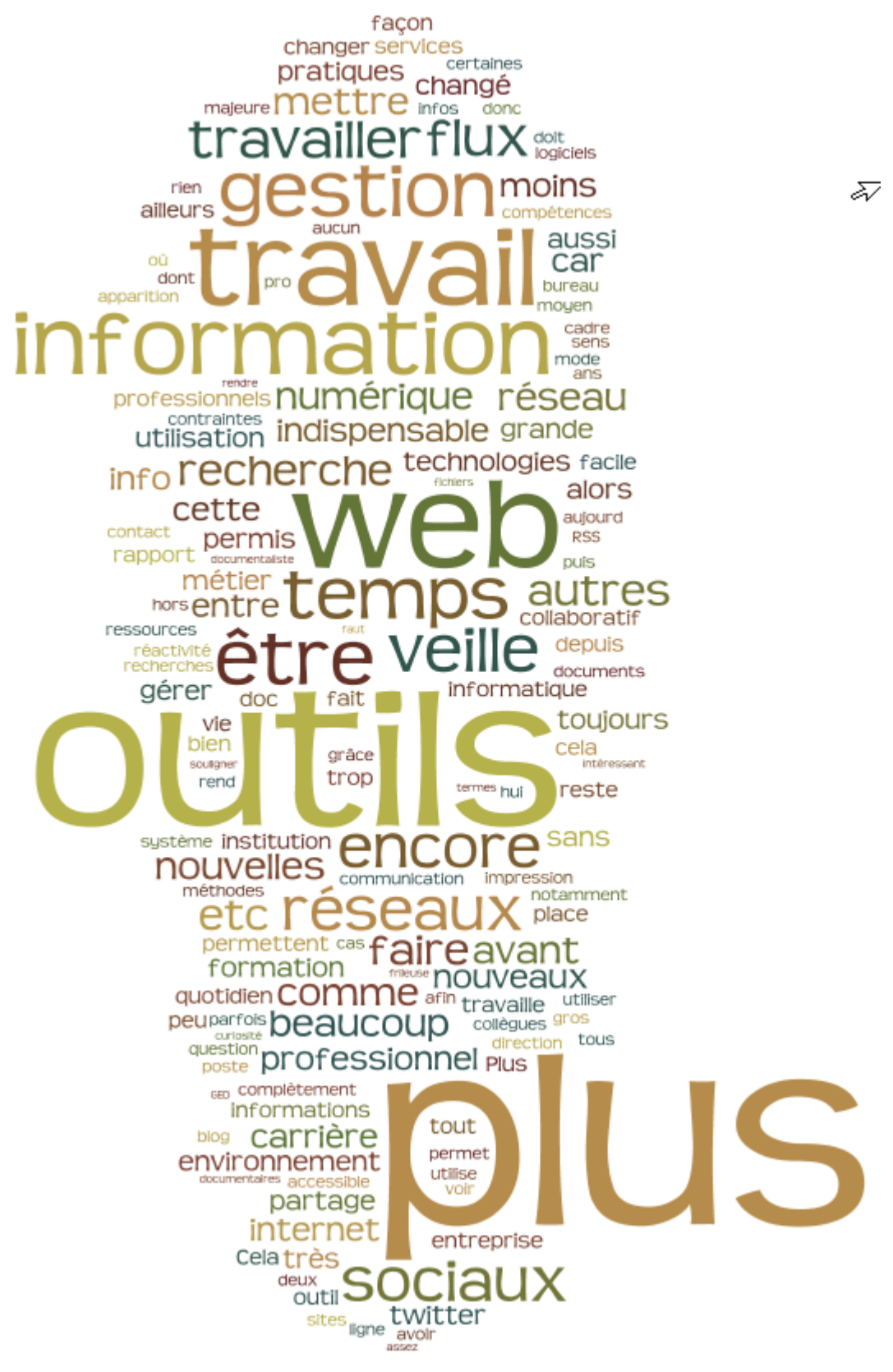

Figure 3. Visualisation wordle des réponses libres.

La forte influence des outils de web 2.0apparaît nettement centrale. Il est aussi étonnant de voir nettement apparaître l'adverbe «plus » ${ }^{6}$ qui démontre cette impression que les outils ne font qu'accroitre le travail et le temps passé à gérer des sources d'informations sans cesse croissante.

\section{Quelle définition du travail ?}

Si le $P K M$ semble a priori répondre aux objectifs à la fois de l'entreprise et du travailleur du savoir, il nous semble que le degré d'indépendance du travailleur du savoir va de pair avec sa capacité à continuer à s'auto-former et à obtenir une reconnaissance au sein de communautés extérieures à

${ }^{6}$ L'adverbe est en fait souvent utilisé pour démontrer la nécessité de disposer de «plus de temps » et souvent pour constater qu'il y a «plus d'informations » à gérer et donc «plus de travail ». 
l'entreprise. Or, cette indépendance et reconnaissance nécessite du temps.

Les travailleurs du savoir peuvent donc ainsi mélanger leurs professions et leurs loisirs ce qui constitue évidemment pour leurs employeurs un élément intéressant. Outre que ce travail supplémentaire n'est pas toujours valorisé, cela n'est pas sans poser de questions sur les conditions de travail et les problèmes familiaux et sociaux qui peuvent en résulter avec l'impression de ne jamais pouvoir débrancher, d'être sans cesse dans le flux ${ }^{7}$. Néanmoins, il s'avère que ces pratiques sont relativement appréciées par des travailleurs qui ont l'impression de prendre ainsi des distances avec leur entreprise ou tout au moins avec un lieu de travail trop cloisonné. Une étude du cabinet Forrester $^{8}$ qualifie de mobile wannabes, ces employés qui utilisent leur smartphonepour améliorer leur productivité. Notre enquête révèle toutefois que le smartphone arrive nettement derrière l'ordinateur portable, le bloc note ou l'agenda papier et la clef usb en tant qu'outil indispensable dans notre enquête.

La démarche de PKMrepose donc initialement sur une double satisfaction, à la fois celle de l'individu qui gagne en indépendance dans un marché du travail fluctuant et concurrentiel, mais également celle de l'organisation qui bénéficie alors de salariés et d'employés plus performants et qui réinvestissent plus facilement leurs acquis extérieurs. Il demeure que cette vision des choses est encore loin d'être partagée et ne présente guère d'effets «sonnants et trébuchants » pour le moment (tableau 1).

\begin{tabular}{|l|l|}
\hline $\begin{array}{l}\text { Ces outils et stratégies de gestion de l'information vous ont-ils fait acquérirune } \\
\text { forme d'indépendance par rapport à vos employeursgrâce au développement de } \\
\text { nouvelles compétences? }\end{array}$ \\
\hline $\begin{array}{l}\text { Oui via une valorisation et une reconnaissance extérieure à votre organisme ou } \\
\text { entreprise }\end{array}$ & $21.52 \%$ \\
\hline Votre expertise acquise n'est pas reconnue par vos employeurs ou clients & $10.93 \%$ \\
\hline Oui mais de manière ponctuelle sans incidence salariale ou hiérarchique & $48.68 \%$ \\
\hline Nsp & $13.91 \%$ \\
\hline Autre & $3.64 \%$ \\
\hline
\end{tabular}

Tableau 1.La reconnaissance des employeurs

\subsection{Une distinction loisir/travail qui devient floue}

Une gestion optimale du PKMréclame souvent beaucoup de temps notamment via des stratégies de veille, de productions d'écrits via les listes de diffusion, les réseaux, les blogs, etc. De même, la construction de cette indépendance voire de formes de reconnaissance est longue et peut nécessiter si ce n'est plusieurs mois, probablement plusieurs années, sans compter qu'il s'agit clairement d'une démarche de formation tout au long de la vie qui nécessite de ne pas rester trop longtemps en dehors

\footnotetext{
${ }^{7}$ Des remarques proches de celles qui sont faites à l'égard des jeunes générations qui privilégient des formes d' « interruption ambiante. »

$8 \quad$ McGillicuddy, SEmployees using their own mobile devices are a growing challenge.<http://searchmobilecomputing.techtarget.com/news/article/0,289142,sid40_gci1335640,00.html>
} 
« du coup ».

Si de prime abord, les travailleurs du savoir peuvent paraitre gagnants, rien n'en est moins sûr. En effet, si l'impression de travailler de manière plus indépendante prédomine, il est tentant de rejoindrel'analyse d'Alan Liu (LIU, 2004) qui y dénonce une forme de culture organisationnelle et informationnelle qui au final aboutit à la fin d'une dichotomie: celle du loisir et du travail.

Le chercheur américain dénonce cette attitude qu'il juge quasi majoritaire parmi les travailleurs du savoir qui consistent à se laisser imposer les lois du «cool ${ }^{9}$ :

"Coolest le point de fuitetechno-informatiquede l'esthétique contemporaine, la psychologie, moralité, la politique, la spiritualité et tout. Finie la beauté, le sublime, la tragédie, la grâce ou le mal: seulement cool ou pas cool»

Tout semble se mélanger et se confondre dans une recherche du cool devenue quasi «naturelle » et qui a même fini par s'imposer dans le monde de la recherche et le milieu des enseignants-chercheurs selon Liu. L'opposition se situant non plus entre travail et loisirs mais entre cool et non cool.

Nous avons donc tenté de vérifier vianotre enquête si la distinction demeurait nette entre les loisirs et le travail un peu à l'instar de ce qui se passe entre vie privée et vie publique avec le numérique. Plus d'un tiers des répondants admettent que cette distinction n'est pas toujours évidente (figure 4). Certes ils ne sont pas majoritaires mais leur proportion n'est pas négligeable. Ces derniers constatent même que leurs activités domestiques sont difficiles à définir clairement. En effet, plus de $70 \%$ ne distinguent plus ce qui relève vraiment des loisirs et du professionnel lorsqu'ils travaillent à domicile.

\section{Avez-vous parfois du mal à distinguer ce qui relève du travail et de la sphère des loisirs ?}

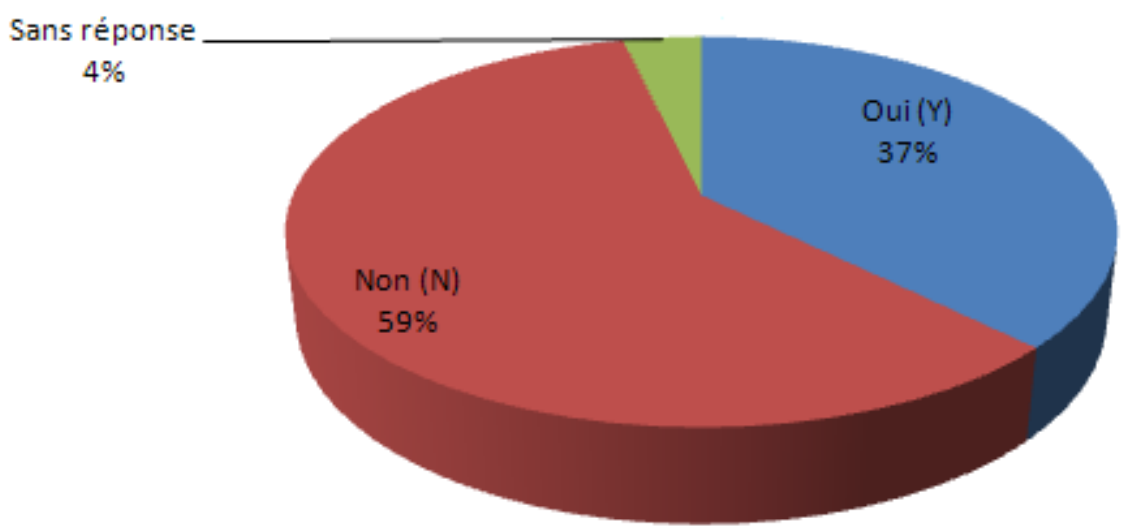

Figure 4. La distinction entre travail et loisirs.

\footnotetext{
${ }^{9}$ Citation originale: "Cool is the techno-informatic vanishing point of contemporary aesthetics, psychology, morality, politics, spirituality and everything. Nor more beauty, sublimity, tragedy, grace or evil : only cool or not cool". In Alan Liu. Idem. p.3
} 
Cette opposition entre les deux groupes (ceux qui distinguent bien travail et loisir et ceux qui ont du mal à distinguer)peut s'expliquer par la façon dont ils considérent le travail. Ceux qui effectuent une césure nette déclarent principalement travailler pour leurs employeurs ou clients. Or ce sentiment est totalement différent pour ceux qui ne distinguent plus loisirs et travail (tableau 2).La césure dans la représentation du travail est très nette :ceux qui ont des difficultés de distinction entre travail et loisirs considèrent majoritairement qu'ils travaillent d'abord pour eux. Il n'est pas non plus étonnant de constater qu'ils aiment davantage travailler chez eux (tableau 5). ${ }^{10}$

\begin{tabular}{|c|r|r|r|r|r|}
\hline \multicolumn{6}{|c|}{ Avez-vous le sentiment de travailler ... } \\
\hline Type de répondants & $\begin{array}{c}\text { Surtout } \\
\text { D'abord } \\
\text { pour vous } \\
\text { pour votre } \\
\text { employeur } \\
\text { ou vos } \\
\text { clients }\end{array}$ & NSP & Autre & Total \\
\hline $\begin{array}{c}\text { Réponses de ceux qui ont du } \\
\text { mal à distinguer travail/loisirs }\end{array}$ & 59 & 30 & 14 & 10 & 113 \\
\hline $\begin{array}{c}\text { Réponses de ceux qui } \\
\text { distinguent travail et loisirs }\end{array}$ & 46 & 104 & 16 & 11 & 177 \\
\hline total & 105 & 134 & 30 & 21 & 290 \\
\hline
\end{tabular}

Tableau 2. Le travail pour soi ou pour les autres

Nous avons procédé au test du khi2 pour vérifier notre hypothèse basée sur l'existence d'une différence de comportements voire d'usages en ce qui concerne les personnes qui ne distinguent plus nettement travail et loisir avec ceux qui opèrent la distinction. Les résultats du test du khi-deux (tableaux 3 et 4) montrent une très forte dépendance entre le sentiment de travailler d'abord pour soi et le fait d'avoir du mal à distinguer les sphères loisir et travail (moins de 1,38489E-06 de risque d'erreur de se tromper en rejetant le risque d'indépendance).

\begin{tabular}{|c|r|r|r|r|r|}
\hline \multicolumn{7}{|c|}{ Avez-vous le sentiment de travailler ... } & Total \\
\hline Type de répondants & $\begin{array}{c}\text { Surtout } \\
\text { D'abord } \\
\text { pour vous }\end{array}$ & $\begin{array}{c}\text { foutre } \\
\text { employeur } \\
\text { ou vos } \\
\text { clients }\end{array}$ & NSP & Autre & 113 \\
\hline $\begin{array}{c}\text { Réponses de ceux qui ont du } \\
\text { mal à distinguer travail/loisirs }\end{array}$ & 40,91 & 52,23 & 11,68 & 8,18 & \\
\hline $\begin{array}{c}\text { Réponses de ceux qui } \\
\text { distinguent travail et loisirs }\end{array}$ & 64,09 & 81,78 & 18,31 & 12,82 & 177 \\
\hline total & 105 & 134 & 30 & 21 & 290 \\
\hline
\end{tabular}

\footnotetext{
${ }^{10}$ Deux réponses maximum étaient toutefois autorisées avec possibilité de préciser le choix et de l'expliciter de manière libre ce qui explique que les résultats sont légèrement supérieurs à $100 \%$. Une des réponses mentionnait d'ailleurs le fait que son lieu de travail était aussi sa maison.D'autres ont argué, que le lieu le plus adéquat variait fortement selon la mission.
} 
Tableau 3

\begin{tabular}{|c|r|r|r|r|r|}
\hline \multicolumn{5}{|c|}{ Avez-vous le sentiment de travailler ... } & Total \\
\hline Type de répondants & $\begin{array}{c}\text { Surtout } \\
\text { D'abord } \\
\text { pour vous } \\
\text { pour votre } \\
\text { employeur } \\
\text { ou vos } \\
\text { clients }\end{array}$ & NSP & Autre & \\
\hline $\begin{array}{c}\text { Réponses de ceux qui ont du } \\
\text { mal à distinguer travail/loisirs }\end{array}$ & 7,995 & 9,450 & 0,456 & 0,403 & 18,305 \\
\hline $\begin{array}{c}\text { Réponses de ceux qui } \\
\text { distinguent travail et loisirs }\end{array}$ & 5,104 & 6,0334 & 0,291 & 0,257 & 11,6861 \\
\hline khi-2 & 13,099 & 15,4841 & 0,748 & 0,661 & 29,992 \\
\hline
\end{tabular}

Tableau 4. Valeurs du Khi-deux

\begin{tabular}{|c|c|c|c|c|c|c|}
\hline \multicolumn{7}{|c|}{ Travaillez-vous mieux ... } \\
\hline \multicolumn{3}{|c|}{ Total des répondants } & \multicolumn{2}{|c|}{$\begin{array}{l}\text { Réponses de ceux qui ont } \\
\text { du mal à distinguer } \\
\text { loisirs/travail }\end{array}$} & \multicolumn{2}{|c|}{$\begin{array}{c}\text { Réponses de ceux qui } \\
\text { distinguent travail et } \\
\text { loisirs }\end{array}$} \\
\hline Réponse & Décompte & Pourcentage & Décompte & Pourcentage & Décompte & Pourcentage \\
\hline Au travail & 208 & $68.87 \%$ & 72 & $63.72 \%$ & 131 & $73.60 \%$ \\
\hline A la maison & 103 & $34.11 \%$ & 52 & $46.02 \%$ & 49 & $27.53 \%$ \\
\hline $\begin{array}{c}\text { Dans un } \\
\text { autre endroit }\end{array}$ & 17 & $5.63 \%$ & 8 & $7.08 \%$ & 9 & $5.06 \%$ \\
\hline
\end{tabular}

Tableau 5. Les lieux de travail préférés.

Nous notons que de la même manière, ceux qui font le moins la distinction sont davantage des utilisateurs des réseaux de signets sociaux type delicious ${ }^{11}$ ou diigo ${ }^{12}$ : plus de $56 \%$ les utilisent de manière régulière voire quotidiennement. Ces outils sont aussi pratiques car ils permettent de retrouver ses données quel que soit l'endroit où on se trouve du moment que l'on dispose d'une connexion internet. Les outils de type web 2.0 participent donc de cet éclatement des lieux de travail. Les possibilités de cloudcomputing, comme potentialités de stockage de données et d'applications en ligne, vont continuer d'influer sur lesreprésentations du travail aussi bien pour les individus que pour les organisations.

Quelle représentation du travail?

Ce qui relève du travail n'est pas clairement perçu de la même façon par les employeurs et les employés. Le travail de veille et de lectures d'informations pour se tenir au courant n'est guère pris en compte par les entreprises. Or, ces aspects requièrent un temps important qui s'effectue souvent à

\footnotetext{
${ }^{11}<$ http://www.delicious.com »

${ }^{12}<\mathrm{http}: / /$ www.diigo.com>
} 
domicile :

«Cela demande un travail assez important et parfois hors du cadre professionnel afin de mettre en place des outils comme Netvibes, lorsque les produits finaux (newsletter, plate-forme collaborative)ne sont pas encore produits, la lecture de blogs ou d'autres commentaires de réseaux sociaux ne sont pas encore perçus comme des activités dites professionnelles par l'entreprise »

L'explication tient au fait que le travail est perçu par l'entreprise comme la mise à disposition au profit de l'organisation ou de l'entreprise de la force de travail de l'individu pour réaliser une mission précise qui correspond au profil de recrutement. Le travail correspond dès lors à une forme de de pénibilité qui rappelle l'étymologie du mot travail qui vient de tripalium : ce triple pieu qui était un instrument de torture. Or le travail doit en fait être différencié de l'emploi. En effet, travailler c'est d'abord travailler pour soi, tandis que la référence à l'emploi implique de mettre sa force de travail au service d'un tiers. Il s'agit d'exprimer le fait que le travailleur, même s'il est employé doit pouvoir poursuivre son individuation sans être aliéné par sa relation avec son employeur. Finalement, l'otium des romains que nous traduisons parfois par loisir, correspond davantage à cette perspective de travail de soi, d'amélioration de soi.Certains usagers mettent clairement en avant cette possibilité de progresser voire de s'auto-former en utilisant les potentialités des outils numériques:

"Pour le travail Le outils web 2.0 me sont d'une grande utilité quotidienne. Je les considère presque comme un moyen d'autoformation. "

Cependant cette recherche pour améliorer son système de gestion de l'information doit être régulièrement revue et améliorée au gré de l'arrivée de nouveaux dispositifs.

"Mes méthodes de travail changent au gré de l'arrivée des nouvelles technologies. Actuellement je ne suis pas totalement satisfaite de la gestion de mon environnement de travail et cherche encore des solutions. »

Dès lors, l'écart se creuse entre ceux qui continuent leur autoformation et qui souhaitent pouvoir réinvestir leurs compétences dans l'organisation et ceux qui au contraire demeurent sur une vision classique du travail avec des horaires et des lieux définis. Une division qui pose de plus en plus de problème au niveau managérial dans les organisations :

"Je tiens à souligner le fait que la prise en main des outils de PKM accentue encore plus les différences au sein des équipes où une partie des professionnels ne font pas l'effort de changer leurs méthodes de travail. La responsabilité est avant tout personnelle, mais doit être imputée au niveau des managers (...)»

Le travail est donc plutôt perçu comme pénible chez ceux qui opèrent nettement la distinction avec les loisirs, tandis que chez ceux qui ont du mal à séparer les deux voient aussi le travail comme un moyen de s'accomplir personnellement. Cette progression personnelle nous semble pouvoir constituer un moteur important dans une démarche de gestion de connaissances et des moyens de partager des savoirs et des savoir-faire tel que c'est d'ailleurs le cas sur les réseaux de loisirs créatifs (Le Deuff, 2010).Cela implique d'envisager à la fois de nouveaux modes de gestion des connaissances ainsi que de nouveaux modes de management des organisations. Cela signifie également repenser nos manières de travailler et laisser plus de place à l'initiative individuelle pour améliorer le collectif :

«Les outils du web 2.0 m'ont donné l'idée de créer un espace collaboratif au sens de ma direction pour changer nos modes de travail. Ce changement s'amorce tout doucement... » 


\section{Conclusion}

Repartir de l'échelon individuel implique une manière différente de concevoir les relations de travail ainsi que les relations avec les objets techniques. D'emblée il est important de ne pas confondre travail et emploi. En effet, travailler c'est d'abord travailler pour soi, tandis que la référence à l'emploi implique de mettre sa force de travail au service d'un tiers. Par conséquent, envisager un réseau personnel d'information et de connaissances implique autant un travail sur soi qu'une possibilité d'employabilité.

Ce travail qui repose sur l'échelon individuel n'est pas antinomique avec des dispositifs d'intelligence collective (Lévy, 1994) tels que ceux qui peuvent être mis en place dans les systèmes de gestion des connaissances.Le terme de connaissances pouvant autant convenir à définir des savoirs que des relations humaines. Cependant, cette réussite potentielle implique un travail de formation individuelle quasi permanent qui influe sur la définition du travail et ses frontières avec la sphère des loisirs.

Le $P K M$ s'inscrit dans un mouvement émergent dont il est encore difficile de prédire les prochaines étapes. Mais il nous parait évident que ceux qui ont adopté un environnement informationnel etde travail optimisé ont changé leur manière de considérer le travail. Si certes, il faut parfois s'inquiéter de nouvelles formes de travail dissimulées au prétexte des «lois du cool », des éléments intéressants sont aussi à porter au profit du travailleur qui gagne davantage à travailler aussi pour lui. De plus, face à cette logique de flux, il faut se concentrer sur des élément stables et notamment les compétences informationnelles mais également les notions et concepts qui peuvent être enseignés, transmis et réinvestis. Les possibilités de construction d'une réelle gestion collective des connaissances ne peuvent se réaliser qu'avec les possibilités d'individuation qui reposent sur le travail en tant que travail sur soi mais également sur la skholé (Le Deuff, 2010b), en tant que capacité de distance critique et de concentration sur un document ou un sujet d'études pendant un laps de temps conséquent.Cela signifie qu'il convient de repenser le rapport aux objets techniques qui constituent l'environnement informationnel et communicationnel des travailleurs du savoir non pas en considérant qu'il s'agit d'un ordre naturel des choses mais comme espace de co-création facilitant notamment l'échange et la création de savoirs. D'où l'importance soulignée par Simondon (Simondon, 1989) de la transmission d'une culture technique pour éviter les mécanismes de dépossession qui ont fait de l'homo faberun homo laborans (Arendt, 1983), c'est-à-dire un travailleur dépossédé de la compréhension de son milieu.

\section{Références}

Arendt, H. (1983)La condition de l’homme moderne. Calmann-Lévy, coll. « Pocket Agora »

Deschamps,Christophe(2009) Le nouveau management de l'information. La gestion des connaissances au coeur de l'entreprise 2.0. FYP éditions

Grant, R. M. (1996)"Toward a knowledge-basedtheory of the firm", Strategic Management Journal, 17 (Winter Special Issue), 109-122.

Huber G. (1990)"A theory of the effects of advanced information technologies on organizational design, intelligence, and decision making”, Academy of Management Review, 15(1). 
Kennedy, P. (2006) Naissance et déclin des grandes puissances. Payot

Le Deuff, O. (2010a) «Réseaux de loisirs créatifs et nouveaux mode d'apprentissage», Distances et savoirs. Vol.8, ${ }^{\circ} 4, \mathrm{p} .601-621$

Le Deuff, O. (2010b) «La skholé face aux négligences : former les jeunes générations à l'attention »,Communication \& Langages n¹63, mars 2010, p.47-61

Le Deuff, O. (2009)La culture de l'information en reformation. (sous la dir. d'Yves Chevalier). Thèse de doctorat en sciences de l'information et de la communication. Université Rennes 2, Septembre 2009

Lévy, L. (1994)L'Intelligence collective: pour une anthropologie du cyberspace. Paris, La découverte

Liu, A. (2004). The Laws of Cool: Knowledge Work and the Culture of Information University Of Chicago Press

Nonaka, I., Takeuchi, H. (2006) The Knowledge-Creating Company: How Japanese Companies

Create the Dynamics of Innovation (p. 304). Oxford UniversityPress, USA.

Pollard, D. (2008)PKM: A bottom-up approach to knowledge management. In Knowledge

Management in Practice: Connections and Context, ed. T.K. Srikantaiah and M.E.D. Koenig, Information Today, pp. 95-114

Raman M. (2006)"Wiki as a 'free' collaborative tool within an organizational setting”, Information Systems Management, Fall, 59-66.

Simondon, G. (1989)Du mode d'existence des objets techniques. Paris, Aubier

Wagner C. (2004) "Wiki: A Technology for Conversational Knowledge management and Group Collaboration", Communications of AIS, Vol. 13, 265-289.

Wenger, E.(1998) Communities of Practice: Learning, Meaning, and Identity. Cambridge University Press

Wright, K. (2005) Personal knowledge management: supporting individual knowledge worker performance. Knowledge Management Research \& Practice 3, 156-165 (1 August 2005) 